\title{
Plasmodium berghei induced priming in Anopheles albimanus independently of bacterial co-infection
}

Jorge Contreras-Garduño ${ }^{1,2 \pi}$, María Carmen Rodríguez ${ }^{11 I}$, Salvador Hernández Martínez ${ }^{1}$, Jesús Martínez Barnetche $^{1}$, Alejandro Alvarado ${ }^{1}$, Javier Izquierdo ${ }^{1}$, Antonia Herrera ${ }^{1}$, Miguel Moreno-García ${ }^{1}$, Maria Elena Velazquez-Meza ${ }^{1}$, Veronica Valverde ${ }^{1}$, Rocio Argotte-Ramos ${ }^{1}$, Mario Henry Rodríguez ${ }^{1}$ and Humberto LanzMendoza $^{1}{ }^{*}$

${ }^{1}$ Centro de Investigación Sobre Enfermedades Infecciosas, Instituto Nacional de Salud Pública, Av. Universidad 655, C. P. 62508. Cuernavaca, Morelos, México.

${ }^{2}$ Departamento de Biología, División de Ciencias Naturales y Exactas, Universidad de Guanajuato. Noria Alta s/n, Noria Alta, 36050. Guanajuato, Guanajuato.

II These authors contributed equally to this work.

*Corresponding authors: humberto@insp.mx; mhenry@ correo.insp.mx 


\begin{abstract}
Priming in invertebrates is the acquired capacity to better combat a pathogen due to a previous exposure to sublethal doses of the same organism. It is proposed to be functionally analogous to immune memory in vertebrates. Previous studies with Anopheles gambiae mosquitoes provide evidence that the inhibitory response to a second challenge by the malaria parasite Plasmodium berghei resulted from a sustained activation of hemocytes by midgut bacteria. These bacteria probably accessed the haemolymph during a first aborted infection through lesions produced by parasites invading the midgut. Since the mosquito immune responses to midgut bacteria and Plasmodium overlap, it is difficult to determine the priming responses of each. We herein document priming induced in the aseptic An. albimanus midgut by $P$. berghei, probably independent of the immune response induced by midgut bacteria. This idea is further evidenced by experiments with $\mathrm{Pbs} 25-28$ knock out parasites (having an impaired capacity for invading the mosquito midgut) and dead ookinetes. Priming protection against a homologous challenge with $P$. berghei lasted up to 12 days. There was greater incorporation of 5-bromo-2'-deoxyuridine into midgut cell nuclei (indicative of DNA synthesis without mitosis) and increased transcription of $h n t$ (a gene required for the endocycle of midgut cells) in primed versus unprimed mosquitoes, suggesting that endoreplication was the underlying mechanism of priming. Moreover, the transcription of hnt and antimicrobial peptides related to an anti-Plasmodium response (attacin, cecropin and gambicin) was enhanced in a biphasic rather than sustained response after priming An. albimanus with $P$. berghei.
\end{abstract}




\section{Introduction}

Priming provides invertebrates with the capacity to produce an enhanced protective response to a microbial invader after being previously exposed to a sub-lethal dose of the same organism (Boman and Steiner, 1981; Kurtz, 2005; Kurtz and Franz, 2003; Little and Kraaijeveld, 2004; Moret and Siva-Jothy, 2003; Sadd and Schmid-Hempel, 2006). Although the underlying mechanisms of priming induction are still unknown, it is considered an evolutionary convergence functionally analogous to immune memory in vertebrates (Hoffmann and Hoffmann, 1990). For this to hold true, a biphasic rather than sustained response is expected in primed invertebrates (for rationale, see (Brehélin, 2008; Chen and Laurence, 1985)). That is, the response induced after the first encounter with a pathogen is followed by an eventual return to basal levels, after which a greater response is produced with a second challenge by the same microorganism.

The report on copepod Macrocyclop salbidus (Kurtz \& Franz, 2003) spurred research that has provided evidence of immune priming for various invertebrate species. However, the induction of priming in mosquitoes by plasmodial infections is still controversial. Since the transmission of Plasmodium, the malaria parasite, to its vertebrate host is dependent on its successful development in the mosquito vector, the possible existence of immune priming in this vector may have important implications for combatting malaria.

After Plasmodium mobile ookinetes invade the midgut epithelium of the mosquito vector, they develop within oocysts on the external surface of this organ. Later Plasmodium attains the final infective stage (sporozoites) upon reaching the salivary glands. Throughout the processes involved from the initial invasion to the sporozoite stage, Plasmodium confronts many potential treats. Among these are the effects of constitutive and induced products of the mosquito immune system (Hillyer et al., 2007; Marois, 2011; Michel and Kafatos, 2005).

Plasmodium coexists with several bacterial species within the aggressive environment of the mosquito midgut (Pumpuni et al., 1996), and the immune genes activated by bacterial and plasmodial infections overlap. For example, transcription factors, PRRS, and antimicrobial peptides induced by bacteria (Dong et al., 2009; Meister et al., 2005; Warr et al., 2008) depict anti-Plasmodium effects. Nevertheless, at least a certain degree of specificity is evidenced by differential gene activation in response to bacterial and plasmodial infections (Baton 
et al., 2009), as well as to different bacterial species (Aguilar et al., 2005; Oduol et al., 2000) and distinct malaria parasite species (Aguilar et al., 2005; Dimopoulos et al., 2002; Dimopoulos et al., 1997; Dimopoulos et al., 1998; Dong et al., 2006a; Dong and Dimopoulos, 2009; Hillyer et al., 2007; Oduol et al., 2000; Tahar et al., 2002; Warr et al., 2008). Furthermore, the induction of the IMD pathway, which signals a potent anti-P. falciparum activity in Anopheles gambiae mosquitoes, is independent of resident midgut microbiota (Garver et al., 2012).

In a previous study, Anopheles gambiae was exposed to an aborted infection with $P$. berghei. After a second challenge, fewer $P$. berghei oocysts were produced in this mosquito species compared to the number of parasites found in control (not previously exposed) mosquitoes. However, the reduction of midgut bacteria by previous antibiotic treatment prevented the protective response, and the researchers concluded that a haemocyte-sustained response to the midgut microbiota (that reached the insect haemocoel during the parasite invasion of the midgut) indirectly reduced the parasite load upon challenge (Rodrigues et al., 2010). Whereas this conclusion seems to be applicable to An. gambiae, the immune response of An. albimanus to Plasmodium probably is distinct.

The aim of the present study was to assess the immune response of An. albimanus to parasites versus bacteria. We exposed aseptic mosquitoes (treated with antibiotics) to aborted infections using live P. berghei gametocytes, both normal and double knockout (Pbs 21, Pbs28) (Tomas et al., 2001), as well as dead P. berghei ookinetes. After a second challenge with the same parasite, fewer oocysts were produced and the mosquitoes showed a biphasic antimicrobial peptide response in all of these groups, indicating that the priming response was directly induced by the parasite. The enhanced induction of endoreplication (DNA synthesis without mitosis) in the midgut and the increased expression of $h n t$, a gene required for a cell's endocycle (Sun and Deng, 2007), afford further evidence for an anti-Plasmodium response and suggest a possible mechanism for priming.

\section{Materials and Methods}




\subsection{Mosquitoes}

Three- to five-day-old An. albimanus were taken from the Tapachula colony (Chan et al., 1994) maintained at the insectary of the National Institute of Public Health (in Cuernavaca, the state of Morelos, Mexico). Mosquitoes were maintained under standardized conditions $\left(80 \% \mathrm{RH}\right.$ and $\left.28^{\circ} \mathrm{C}\right)$ and fed ad libitum with $5 \%$ sucrose.

\subsection{Mosquito antibiotic treatment}

Tryptic Soy Agar and Blood Agar cultures of midguts from a sample of these An. albimanus mosquitoes revealed infection with Enterococcus durans hirae and Serratia marcescens. These bacteria were susceptible to penicillin, streptomycin and gentamicin (gender/species and antibiotic susceptibility was identified with Microscan ${ }^{\circledR}$ ). Accordingly, cotton pads soaked in 5000 U of penicillin, $5 \mathrm{mg} / \mathrm{ml}$ of streptomycin, $10 \mathrm{mg} / \mathrm{ml}$ neomycin (Sigma), and $50 \mu \mathrm{g} / \mathrm{ml}$ Gentamicin in sterile 5\% sucrose were offered to mosquitoes (beginning at eclosion) throughout the experiments. The midgut bacteria that remained in a sample of antibiotic-treated mosquitoes were quantified in duplicate with Triptic Soy Agar and Blood Agar at $24 \mathrm{~h}$ after both the first (priming) blood meal and the challenge with GFP ookinetes. Quantification of bacteria was done using RT-PCR cDNA from midgut samples and universal bacterial primers to amplify 16SrRNA (Fw: 5'TCCTACGGGAGGCAGCAGT-3' and Rv: 5' ACTACCAGGGTATCTAATCCTGTT-3'). Bacteria were normalized using An. gambiae ribosomal protein S7 as an internal standard, as described by Rodrigues et al. (2010).

\subsection{Parasite preparations}

A gametocyte-producing Plasmodium berghei strain (ANKA 2.34, kindly donated by Robert E. Sinden, Imperial College, UK) was used. Mice were infected with Plasmodium berghei gametocytes as previously described (Rodriguez et al., 2002). Briefly, BALB/c male mice were infected intraperitoneally with approximately $1 \times 10^{6}$ parasites. On day three, their gametocytaemia was estimated in Giemsa-stained tail blood smears. Only mice with a gametocytaemia between 3-5\% were used. Ookinetes were prepared as previously 
described (Rodriguez et al., 2002). Briefly, gametocyte-infected mouse blood was diluted 1:5 with RPMI-1640 culture medium (20\% heat inactivated fetal bovine serum at $\mathrm{pH} 8.3$ ), passed through a CF-11 column (cellulose powder), and incubated at $20-21^{\circ} \mathrm{C}$ for $24 \mathrm{~h}$.

\subsection{Priming infections}

Antibiotic-treated An. albimanus were fed with normal blood (unprimed control groups) or P. berghei gametocyte-infected mouse blood (primed groups). A group of mosquitoes untreated with antibiotics was added in these initial experiments. Mosquitoes (kept in groups) were allowed to feed for one hour at room temperature. After removing unfed mosquitoes, the engorged ones were placed in an incubator at $20-21{ }^{\circ} \mathrm{C}$ for two days in order to allow for parasite development. After $24 \mathrm{~h}$ of incubation, ookinetes were observed in the blood meal bolus in $80 \%$ of a sample of engorged mosquitoes. All mosquito groups were provided a cotton pad soaked with $8.0 \%$ fructose containing $0.05 \%$ para-aminobenzoic acid (PABA). To interrupt parasite development, mosquitoes were moved to a room with a temperature of $28{ }^{\circ} \mathrm{C}$ on day three and remained there until challenged. The analysis of midguts taken from a sample of mosquitoes dissected on day 15 post-infection and then stained with mercurochrome (Eyles, 1950) indicated that these mosquitoes did not develop oocysts (aborted infection).

To investigate the effect of non-invasive parasites and reduce the possibility of bacteria invading the mosquito midguts, mouse blood was infected with gametocytes of double knockout (DKo) P. berghei (kindly provided by Robert E. Sinden, Imperial College, UK), lacking Pbs 25 and Pbs 28 (Tomas et al., 2001), to feed experimental mosquitoes. Hence, three mosquito groups were used: those fed with blood meal infected with the original P. berghei ANKA strain $(n=144)$ or with DKo parasites $(n=96)$, and those fed with normal uninfected mouse blood (the unprimed control; $n=139$ ). All of these experiments were performed in triplicate.

Other experiments were performed to eliminate the possible effect of damage to the mosquito midgut epithelium. For this purpose, $P$. berghei ookinetes at an equivalent concentration of $1200-1300$ ookinetes $/ \mu \mathrm{L}$ were killed with a 1 min freeze-thaw cycle using liquid nitrogen and then fed in a blood meal to experimental mosquitoes. The four mosquito groups used in these experiments were fed with blood meal infected with either 
the original $P$. berghei ANKA strain $(n=70)$, dead ookinetes $(n=70)$, or live ookinetes $(n=70)$, or with normal uninfected mouse blood (the unprimed control; $n=70$ ). In all of these experiments, performed in duplicate, mosquitoes were placed at $20-21{ }^{\circ} \mathrm{C}$ for two days to allow $P$. berghei to develop before being moved to a room at $28{ }^{\circ} \mathrm{C}$ to interrupt parasite development. Mosquitoes remained under the latter condition until challenged.

\subsection{Challenge infections}

Unprimed mosquitoes (the control fed with normal mouse blood) and primed mosquito groups were allowed to oviposit. On day seven they were challenged with a second blood meal containing $P$. berghei ookinetes that expressed a green fluorescent protein (GFP) (Franke-Fayard et al., 2004) (provided by R. E. Sinden, Imperial College, UK). Ookinetes were prepared as previously described (Rodriguez et al., 2002). Briefly, ookinete cultures were spun $\left(5 \mathrm{~min}\right.$ at $200 \mathrm{~g}$ and $20^{\circ} \mathrm{C}$ ), counted in $0.5 \mu \mathrm{L}$ smears stained with Giemsa, and adjusted to 1200-1300 parasites/ $\mu \mathrm{L}$ (at 40\% hematocrit) using normal mouse blood depleted of white blood cells by passage through CF11 columns. This blood meal was then offered to mosquitoes using artificial glass feeders (Winger et al., 1988).

After feeding with GFP parasites, mosquitoes were maintained at $20-21{ }^{\circ} \mathrm{C}$ and provided with $8.0 \%$ fructose containing $0.05 \%$ p-aminobenzoic acid (PABA). On day five post-challenge, the insects were dissected and their midguts analyzed under a UV light microscope (Leica DM 1000) to determine the percentage carrying GFP oocysts (prevalence of infection), the number of oocysts per mosquito (intensity of infection), and the intensity of infection taking into account only the infected mosquitoes (the mean intensity of infection).

\subsection{Bacterial inoculations}

To resemble the effect of a possible invasion of the haemocoel by midgut bacteria, experiments were conducted in mosquitoes inoculated with bacteria the day after the priming or challenge infection. Mosquitoes were briefly stunned on ice and inoculated between the fourth and fifth abdominal segments of the pleural membrane (Hernandez-Martinez et al., 2002) with $0.25 \mu \mathrm{L}$ of PBS (Gibco BRL, Grand Island, NY) containing approximately $1 \times 10^{3}$ heat-inactivated E. durans or E. coli (experiments using live bacteria produced very high 
mosquito mortality). Unprimed mosquitoes were inoculated with $0.25 \mu \mathrm{L}$ of PBS or not treated. Glass capillary tubes were used to make inoculation needles using a micro-needle maker P-30 (Sutter Instrument Company, CA, USA). Priming and challenge infections, the latter at 7 days post-priming, were carried out as described above.

\subsection{Evaluation of mRNA expression of antimicrobial peptides}

To analyze the immune response of mosquitoes after the priming/challenge infection, the mRNA expression of antimicrobial peptides (AMPs: attacin, cecropin and gambicin) was determined in midguts as well as abdomen carcasses (the latter consisting of the remaining abdomen wall with fat body, dorsal vessel, etc., after removing the midgut). These AMPs have been previously used as immune markers in midgut and fat body of mosquitoes during Plasmodium infection (Herrera-Ortiz et al., 2011). Twenty midguts and abdomen carcasses were collected from $P$. berghei-primed and unprimed mosquitoes at different times- $-0,6,12,18,24$, and $48 \mathrm{~h}$ and 8 days after the first (priming) exposure; 0, 6, 12, 18, and $24 \mathrm{~h}$ after the second (challenge) exposure. Total RNA was obtained from tissues using the Quick-RNAtm Miniprep kit (Zymo Research) and cDNA was obtained with the Gene Amp kit (Applied Biosystems). QRT-PCR was carried out using the Syber Green I Kit (Applied Biosystems) according to the manufacturer's instructions. To avoid contamination with genomic DNA, tissues were treated with the DNAse enzyme (Invitrogen).

The sequence of primers used was as follows: Gambicin (AGAP008645), RT_Gam_F (CGTGCGATGGTCAGACGAT) and RT_Gam_R (CGCCGCGTTCACAAGAA); attacin (AGAP005620), Atta_F (CGC TAC AAA GGC AAG ATG AAC) and Atta_R (TGT TTC CGC TCG CAC TCT TC); cecropin (AGAP000694), Cec3_F(GAAATTGGCAAACGACGTGAA) and Cec3_R (GCGATGCTAAAAGACTAAGGGC). Actin RT_ActU_R (CGA TCC ACT TGC AGA GCC AGT) and RT_Act3.2_F (TAC GCC AAC ATT GTC ATG TCC). QRT-PCR conditions and primers were the same as previously reported (Herrera-Ortíz et al., (Herrera-Ortiz et al., 2011).

The relative expression was determined by the comparative "delta delta $C t "(\Delta \Delta C t)$ method, employing three replicates per sample. Results are expressed as X P.b./ X n.i., where X P.b. is the mean gene expression level in primed mosquitoes and $\mathrm{X}$ n.i. is the mean gene expression level in unprimed mosquitoes. Comparisons 
were made of the level of expression of AMPs between primed and unprimed midguts and abdomens during the first (priming) and second (challenge) parasite exposures. Results were analyzed using a t-test with Welch correction (Prisma GraphPad software).

\subsection{Analysis of endoreplication}

To investigate whether midgut cells underwent multiplication or DNA synthesis without mitosis, 5-bromo-2'deoxyuridine (BrdU) (Boehringer Mannheim GmbH, Germany) was added to the cotton pad mixture (5\% sucrose at a concentration of $1 \mathrm{mg} / \mathrm{ml}$ and the antibiotics, as described in section 2.2) that was offered to mosquitoes after eclosion and throughout the experiments. Cotton pads containing BrdU and antibiotics were replaced every $24 \mathrm{~h}$.

\subsubsection{Immunofluorescence assays of mosquito midguts after using BrdU}

The presence of mitotic spindles was investigated at 12 and $24 \mathrm{~h}$ after priming or after the blood meal with normal mouse blood (without parasites) in the unprimed group. No mitotic spindle structures were observed at any time, but the first BrdU labeled cell nuclei were observed at $24 \mathrm{~h}$ in both groups (data not shown). Samples of 10 mosquito midguts from primed and unprimed groups, taken every $48 \mathrm{~h}$ after priming and after the challenge, were processed as previously reported (Hernández-Martínez et al., 2013) for immunofluorescence and ELISA assays. Briefly, midguts were dissected and fixed during $2 \mathrm{~h}$ with $4 \%$ paraformaldehyde in PBS. The fixative was removed and the samples were permeated with cool methanol for $10 \mathrm{~min}$, and then washed in PBS containing 1\% Tween-20 (PBST). Samples were hydrolyzed in $2 \mathrm{~N} \mathrm{HCl}$ for $1 \mathrm{~h}$ at $37^{\circ} \mathrm{C}$, neutralized by three changes of Hank's solution, and then washed with PBST. Samples were blocked for $1 \mathrm{~h}$ at $37^{\circ} \mathrm{C}$ with $2 \%$ bovine serum albumin in PBS (PBSA) before being incubated at $4{ }^{\circ} \mathrm{C}$ overnight with a fluoresceine-labeledmouse anti-BrdU monoclonal antibody at 1:100 in PBSA (Roche GmbH Mannheim, Germany). After rinsing in PBST, samples were mounted on slides using FluorSave (Merck KGaA, Darmstadt, Germany) and analyzed using a confocal microscope (C2) coupled to an epi-fluorescent microscope (E-600). Images were recorded using the NIS-Elements C software (all from Nikon, Japan). 


\subsubsection{Relative quantification by ELISA of BrdU in mosquito tissues}

To detect BrdU in midguts that were undergoing DNA synthesis, primed and unprimed samples were obtained as described in section 2.8.1 and processed as previously described (Hernandez-Martinez et al., 2013). DNA from midguts was obtained by phenolic extraction and incubated with $0.2 \mathrm{mg} / \mathrm{ml}$ of RNAse A (Gibco, Grand Island, NY, USA) for $1 \mathrm{~h}$ at $37^{\circ} \mathrm{C}$ (Sambrook and Russell, 2001). DNA samples were suspended in $50 \mu \mathrm{l}$ of PBS containing $0.02 \%$ sodium azide (Sigma, St. Louis, MO, USA). DNA concentration was determined at 260/230 nm in a NanoDrop 1000 spectrophotometer (Thermo Scientific, Wilmington, DE, USA). Ten micrograms of DNA in $50 \mu \mathrm{l}$ of bicarbonate buffer were transferred to pre-treated poly-L-lysine wells of 96well plates (Nalgene Nunc, Naperville, IL, USA), and incubated for $24 \mathrm{~h}$ at $4{ }^{\circ} \mathrm{C}$. Incorporated BrdU was determined by ELISA by using an anti-BrdU-peroxidase-conjugated monoclonal antibody (Roche, GmbH Mannheim, Germany), and O-Phenylenediamine (Zymed, San Francisco, CA, USA) as substrate (Bravo et al., 1987). The absorbance was recorded at $450 \mathrm{~nm}$ in an ELISA plate reader (DAS, Palombara Sabina, Italy). DNA from untreated mosquito midguts (without antibiotics, BrdU, or blood meal) was used as a blank to calibrate the ELISA plate reader. The relative absorbance value of experimental samples was calculated by assigning a value of 1 to the lowest absorbance of unprimed samples (fed with normal mouse blood). Experiments were independently reproduced two times. Differences between mean absorbance values were analyzed using twoway variance (ANOVA) and comparisons between treatments were analyzed using the Bonferroni post-test (GraphPad PRISM 3.03 software).

\subsubsection{Analysis of endocycle-related genes}

To investigate gene expansion in the priming response to $P$. berghei, we determined the induction of Hindsight (hnt1) (Sun and Deng, 2007) in the midguts of primed and unprimed mosquitoes. The putative ortholog of $h n t$ in An. albimanus (Martinez-Barnetche et al., 2012) was identified and its expression analyzed after priming and the challenge with $P$. berghei. Twenty midguts from each group (with and without antibiotics) were obtained at $72 \mathrm{~h}$ after priming and the challenge. Total RNA was extracted from midgut tissues using the TRI Reagent from 
SIGMA and following the manufacturer's instructions. Midgut RNA was used for first strand cDNA synthesis reactions with the Maxima First strand cDNA synthesis kit from Thermo Scientific. The hnt primers used were 5’ GAT CAT ATG CGC CAG TGT CAT 3’ and 5’ATT GTT GCC GCT GCT CT 3’. Real Time PCR was carried out using the Syber Advantage qPCR Premix from Clontech and following the manufacturer's instructions. The PCR reactions were made in a 7500 Fast Real-Time PCR System from Life Technologies. The dynamic range of primers was determined and the amplicon was validated by melting curve analysis, which showed that a single amplification fragment is produced. The cycling conditions were denaturation at $95^{\circ} \mathrm{C}$ for 2 min and 35 cycles of denaturation at $95^{\circ} \mathrm{C}$ for $30 \mathrm{~s}$, annealing at $50{ }^{\circ} \mathrm{C}$ for $30 \mathrm{~s}$, and extension at $72{ }^{\circ} \mathrm{C}$ for 30

s. Change in the relative amount of hnt mRNA was calculated using the comparative CT method (Delta Delta CT) with actin as a normalizing control (Herrera-Ortiz et al., 2011).

\subsection{Statistics}

The prevalence of infection (number of mosquitoes presenting GFP oocysts) and the intensity of infection (number of oocysts per mosquito) were compared between groups in all experiments. AMPs expression was analyzed using an unpaired t-test with Welch correction. The data on intensity of infection and the mean intensity (number of oocysts per midgut including only infected mosquitoes) were analyzed with a nonparametric test (Mann-Whitney U-test; two-tail $P$ value). The numbers of infected and uninfected mosquitoes (prevalence) were analyzed with 2 X 2 tables and an $X^{2}$ test, d.f $=1$. Statistical tests were performed using the Graph Pad software ${ }^{\circledR}$.

\section{Results}

\subsection{Plasmodium berghei-induced priming}

We investigated whether eliminating the midgut bacteria halted the induction of priming in An. Albimanus to $P$. berghei. For this purpose, we compared the induced resistance to infection after an aborted primary infection in two groups of mosquitoes (treated and not treated with antibiotics), as well as the resistance to infection in two 
unprimed mosquito groups that were not exposed to a primary aborted infection (treated and not treated with antibiotics). No bacteria grew in cultures of samples of antibiotic-treated mosquito midguts taken before and after the priming and challenge infections, but traces of bacterial RNA were still detected by RT-PCR (Figure S1). After the challenge and compared to the unprimed group (with 57\% infected mosquitoes), there were differences in the proportion of infected mosquitoes in the primed group without antibiotics $\left(46 \%\right.$ infected; $\chi^{2}=$ $7.28, p=0.0035)$ and the primed group with antibiotics $\left(34 \%\right.$ infected; $\left.\chi^{2}=18.28 ; p<0.0001\right)$. In the control group, treatment with antibiotics led to a lower proportion of infected mosquitoes than the absence of this treatment. The mean number of oocysts/midgut was higher in the unprimed group $(29.6 \pm 2.63)$ than in either of the primed groups, whether treated with antibiotics $(15.40 \pm 2.85 ; p=0.0053)$ or not $(18.64 \pm 2.36 ; p<0.0001)$

\section{(Figure 1).}

\subsection{Mosquito midgut damage is not necessary to induce priming}

Although bacteria were not detected when culturing midgut tissue of antibiotic-treated mosquitoes, there remained the possibility that residual microorganisms still reached the haemocoel during parasite invasion. To further reduce this possibility, we conducted priming experiments with antibiotic-treated mosquitoes exposed to an aborted infection with double knockout (DKo) P. berghei gametocytes lacking Pbs 25 and Pbs 28, which greatly reduces the capability of parasites to invade the midgut epithelium (Tomas et al., 2001). After the challenge in these experiments, the proportion of infected mosquitoes was high in all groups, whether unprimed (81\% infected) or primed with P. berghei gametocytes. The DKo group had $75 \%$ infected mosquitoes $\left(\chi^{2}=\right.$ 0.9938; $p<0.1594)$ and the ANKA strain group had $59 \%\left(\chi^{2}=15.64 ; p<0.0001\right)$. Compared to the unprimed group, the mean number of oocysts/midgut was lower in primed mosquitoes, both with DKo parasites $(6.58 \pm$ 0.90 versus $27.23 \pm 2.71 ; p<0.0001)$ and the ANKA strain $(18.27 \pm 3.27$ versus $27.23 \pm 2.71 ; p<0.0001)$

\section{(Figure 2).}

To completely eliminate midgut damage during ookinete invasion, the effect of priming by dead $P$. berghei ookinetes was compared to that caused by aborted infections with live gametocytes and live ookinetes, as well as to the result with unprimed mosquitoes. After the challenge, the proportion of infected mosquitoes in 
groups primed with live gametocytes ( $55 \%$ infected; $\left.\chi^{2}=5.35 ; p=0.0103\right)$, live ookinetes ( $30 \%$ infected; $\chi^{2}=$ 27.54; $p<0.0001)$ and dead ookinetes $\left(60 \%\right.$ infected; $\left.\chi^{2}=3.27 ; p=0.0352\right)$, was lower than in the unprimed group (75.7\% infected). Also, the intensity of infection (mean number of oocysts/midgut) was lower in mosquitoes primed with $P$. berghei gametocytes ( $7.38 \pm 2.26 ; p<0.0001)$, live ookinetes $(5.28 \pm 2.51 ; p<$ 0.0001), and dead ookinetes $(7.85 \pm 1.55 ; p=0.0002)$ compared to unprimed mosquitoes $(23.52 \pm 4.47)$ (Figure 3).

To simulate the effect of midgut bacteria possibly invading the mosquito haemocoel, priming experiments were conducted $24 \mathrm{~h}$ after the first aborted infection with live ookinetes using antibiotic-treated mosquitoes that were inoculated with heat-killed E. coli or E. durans. In these experiments the intensity of infection after challenge was lower in primed mosquitoes that were not inoculated $(15.12 \pm 2.54)$ than in bacteria inoculated mosquitoes ( $29.47 \pm 4.07$ for E. coli and $30.07 \pm 10.63$ for $E$. durans) and unprimed mosquitoes (28.77 \pm 3.98$)$ (data not shown).

\subsection{Priming with $P$. berghei induced a biphasic transcription of AMPs and Plasmodium-induced peptides}

AMPs have been previously used as immune markers in mosquitoes infected with Plasmodium (Bahia et al., 2011). Moreover, we have reported that attacin, gambicin and cecropin mRNA are induced during the infection of An. albimanus with P. berghei (Herrera-Ortiz et al., 2011). In experiments of the present study with $P$. berghei-primed mosquitoes that were treated with antibiotics, Reverse Transcription real-time PCR (RT-PCR) revealed an increase in the relative mRNA levels in midgut and abdomen carcasses after the priming infection, soon followed by a decrease to basal levels of pre-infection, and again an increase after the challenge reaching higher levels than those observed during the first parasite encounter (Figure 4A and B). This pattern of biphasic kinetics found presently strongly suggests that the mosquito immune response during immune priming is biphasic rather than enhanced. Higher AMP expression was observed in primed than unprimed mosquitoes, but the only significant differences were found in relation to gambicin in primed versus unprimed midguts after the challenge $(p=0.038)$, and gambicin $(p=0.037)$ and cecropin $(p=0.037)$ in primed versus unprimed abdomen carcasses after the challenge. 


\subsection{Primed mosquitoes and endoreplication}

Regarding Drosophila, the high quantity of proteins required for development is produced by a higher quantity of transcripts from an increased number of DNA copies that are produced in successive rounds (endocycle) of DNA duplications without cell mitosis (endoreplication). We previously documented a differential DNA synthesis in An. albimanus tissues induced by an immune challenge with different microorganisms (Hernandez et al., 2013). Here we explored whether this phenomenon was involved in the enhanced response of primed mosquitoes after the challenge.

The incorporation of bromodeoxiuridine into the cell nuclei was observed in both primed and unprimed groups as early as $24 \mathrm{~h}$ after the blood meal (data not shown). The BrdU signal and relative values were similar between groups during several days, but after the challenge they showed significant differences (Figure 5). Twenty four hours after the challenge with $P$. berghei, incorporation of BrdU was greater in primed versus unprimed mosquitoes, as detected by immunofluorescence. Also, the semiquantitative ELISA analysis of BrdU levels indicated a significant increase $(p<0.0001)$ in DNA synthesis in the primed group $24 \mathrm{~h}$ after the challenge (Figure 5B). As no mitosis was observed during either the priming or challenge infection with $P$. berghei, we investigated one key factor that participates in cell cycle switching towards endoreplication in Drosophila (Sun and Deng, 2007) to determine whether it was involved in the production of the priming response in An. albimanus. This key factor is $h n t$, a zinc-finger protein that mediates the role of Notch. First, we identified the putative ortholog of hnt in the An. albimanus transcriptome (Martinez-Barnetche et al., 2012) and analyzed the expression of this gene in antibiotic-treated mosquitoes. Compared to unprimed mosquitoes (fed with normal mouse blood), there was a 5.2-fold upregulation of $h n t$ expression in primed mosquitoes. At seven days post-priming, mosquitoes were challenged with $P$. berghei ookinetes. At $72 \mathrm{~h}$ post-challenge, an 8.1-fold increase in hnt was found in mosquitoes primed with live ookinetes (Figure 5C) and a 19.0-fold increase in mosquitoes primed with dead ookinetes (data not shown).

\section{Discussion}


The reduced development of $P$. berghei in primed An. albimanus found presently is similar to the results of a previous study (Rodrigues et al., 2010) indicating that a first exposure of Anopheles gambiae mosquitoes to $P$. berghei followed by the interruption of parasite development induces a new host state that limits parasite development after a second exposure. Induced priming has been observed in other invertebrates, including insects (Kurtz, 2005). The current results demonstrate for the first time that priming was induced by the parasite independently of the participation of midgut bacteria, thus indicating the existence of separate pathogen recognition mechanisms for bacteria and the Plasmodium parasite. Also for the first time in invertebrates, we documented that the humoral immune response to the priming and challenge infections followed a biphasic rather than sustained pattern, indicating an underlying mechanism akin to immune memory. After induction of the host defense, a broad spectrum of effector responses could explain the previously observed overlap between the anti-bacterial and anti-plasmodial mosquito immune responses (Pike et al., 2014).

Insects respond to bacteria and other pathogens with humoral and cellular innate immune responses (Chen and Laurence, 1985; Gonzalez-Ceron et al., 2003; Hoffmann and Hoffmann, 1990; Noden et al., 2011). Analysis of the mosquito immune response, including the priming effect against Plasmodium, is complicated by the coexistence of midgut bacteria whose presence increases greatly after a blood feeding (Gonzalez-Ceron et al., 2003; Noden et al., 2011). These bacteria directly affect parasite development (Cirimotich et al., 2011) and induce the expression of immune genes whose products can damage Plasmodium (Dong et al., 2009; Meister et al., 2005). Such genes are activated when malaria ookinetes invade the midgut epithelium (Dimopoulos et al., 2002), meaning that the immune response to Plasmodium may actually be triggered by the interaction of bacteria with the parasite-damaged epithelium and/or their invasion of the haemocoel. Thus, in an An. gambiae$P$. berghei model, it was proposed that bacteria invading the midgut during the first parasite encounter activated haemocytes and anti-Plasmodium haemocyte-specific genes (thioester-containing protein TEP1 and leucine rich repeat immune protein LRIM1) (Blandin et al., 2004; Vlachou et al., 2005), that in turn mediated a sustained anti-parasite response for up to 14 days (Rodrigues et al., 2010).

In both the An. gambiae and An. albimanus models (used by Rodrigues and our group, respectively), there were similar strategies employed in the priming experiments that involved aborted infections with live $P$. 
berghei. Whereas the elimination of bacteria arrested the activation of the priming response in An. gambiae, the present study demonstrates that in An. albimanus the induction of priming by $P$. berghei was independent of the possible mechanisms of the immune response for combating midgut bacteria. We detected traces of bacterial RNA by RT-PCR after priming in antibiotic-treated mosquitoes, which indicated that antibiotics did not completely eliminate midgut bacteria. However, the abatement of bacteria reached levels undetectable by culture. Hence, the possibility of bacterial growth and haemocoel invasion was diminished to a minimum.

The enhanced response to the second challenge was also induced in antibiotic-treated mosquitoes by Pbs 21/28-Ko parasites, reducing the possibility that anti-parasite priming was induced by the direct contact of midgut bacteria with the mosquito haemocoel. This result is in line with previous studies showing that $P$. berghei gametocytes in the midgut lumen activate the mosquito immune response (Dimopoulos et al., 2001; Tahar et al., 2002). Furthermore, the exposure of An. albimanus to dead ookinetes in the midgut lumen had a similar effect to that induced by live parasites, confirming the priming effect exerted by a yet unknown parasite component, which acted in a midgut environment where bacteria was abated to a minimum and no epithelium damage was inflicted.

We also sought to test the existence of immune priming triggered by a bacterial invasion of the haemocoel. For this purpose, we conducted experiments using dead E. durans (the most abundant commensal isolated from our An. albimanus colony) and E. coli, but no priming against P. berghei was observed. Recognizing that dead bacteria do not fully represent a proper bacterial infection, we inoculated mosquitoes with live bacteria, which resulted in high mosquito mortality. This indicates that a possible low and controlled infection as a result of multiple midgut epithelial damage by invading parasites is unlikely.

Overall, these observations indicate that an interrupted infection with $P$. berghei directly induced priming. That is, the induced immune response was biphasic rather than sustained and was induced by Plasmodium rather than bacteria. Thus the current results provide evidence that the anti-bacterial immune response could mask the parasite-induced response. The discrepancy between the present results with $A n$. albimanus and those reported by Rodrigues et al. (2010) in relation to An. gambiae seem to indicate a distinct immune response in the two mosquito species. Whereas the immune response of An. gambiae appears to require 
the participation of midgut microorganisms, An. albimanus is able to directly mount priming responses to the parasite. Further research is required to identify the pathways involved in the enhanced response after priming.

Previous studies have found nonspecific and specific priming in other insects (Akira et al., 2001; Kurtz, 2005; Medzhitov and Janeway, 2002; Sadd and Schmid-Hempel, 2006; Schulenburg et al., 2007). Similar to vertebrate immunity, insects and specifically mosquitoes possess a sophisticated repertoire for nonspecific and specific pathogen recognition mediated by diverse immune pathways and effector molecules (Waterhouse et al., 2007). For mosquitoes it has been reported that pattern recognition receptors (PRRs) participate in the activation of the innate immune system through the recognition of the pathogen molecular patterns (PAMPs) of invading microorganisms (Dimopoulos et al., 2002; Dimopoulos et al., 1997; Dimopoulos et al., 1998). At least three signaling pathways that mediate the immune response are conserved in mosquitoes and other insects- that of Toll-like receptors, of Immune Deficiency (IMD), and of the Janus kinase signal transducer and activator of transcription (JAK-STAT) (De Gregorio et al., 2002; Meister et al., 2005; Pike et al., 2014; Sanders et al., 2005). Toll components are active against Gram-positive bacteria, fungi (De Gregorio et al., 2002) and viral infections (Costa et al., 2009; Garver et al., 2009). IMD participates in responses against Gram-positive and Gram-negative bacteria as well as viruses (Garver et al., 2013; Gupta et al., 2009; Meister et al., 2005). Toll, IMD and the JAK-STAT participate in the response against plasmodial infections (Bahia et al., 2011; Cirimotich et al., 2011; Dong et al., 2006b; Pham et al., 2007; Rolff and Siva-Jothy, 2003).

Different PRRs, pathways and effector molecules could provide mosquitoes with some degree of immune specificity. For instance, a family of six Gram-negative binding proteins (GNBPs) functioning as PRRs has been identified in An. gambiae, and it is known that the activation of these by invading pathogens varies. Whereas GNBP4 is activated by a broad range of pathogens, other GNBPs have a more restricted function (Warr et al., 2008). The Down syndrome cell adhesion molecule (Dscam) superfamily with hyper variable immunoglobulin domains (Smith et al., 2011; Witteveldt et al., 2004) provides the An. gambiae innate immune system with over 30,000 possible splice-form specific PRRs. The specificity of mosquito innate immune responses includes the recognition of different malaria parasite species (Ng et al., 2014; Tahar et al., 2002). 
Nevertheless, the specificity that differential PRR activation may provide mosquitoes does not exclude an overlap of the immune responses to distinct types of pathogens. Though not identical, the pattern of genes induced by parasites and bacteria are similar (Aguilar et al., 2005; Dimopoulos et al., 2002; Dimopoulos et al., 1997). For instance, the fibrinogen-related proteins (FREPs, with hyper variable domains) respond to a challenge by bacteria, fungi and Plasmodium, and they present complementary and synergistic functions (Dong et al., 2009). On the other hand, $\alpha$-2-macroglubulin is strongly induced by parasites but scarcely or not induced by bacteria (Kurtz, 2005).

The overlap of the products of various immune mechanisms makes it difficult to discern between pathogen-specific responses. In this sense, a study on An. stephensi reported the upregulation of a wide range of immune genes, including Toll and JNK, by the IMD transcription factor Rel2, thus providing evidence of the interaction between many immune response pathways (Dong et al., 2009). In order to distinguish between the immune response induced by bacteria and malaria parasites, bacteria have been eliminated in insects with antibiotics. After the elimination of midgut microbiota in mosquitoes, for example, it was found that Plasmodium continued to induce defensin and GNBP production (Dimopoulos et al., 2002). Similarly, it has been reported that the participation of IMD pathway components and their regulated effectors (TEP1, APL1 and LRM1) in the control of P. falciparum by An. gambiae is independent of the midgut microbiota (Garver et al., 2012).

Previously, it was observed that along with antibacterial immune genes, specific anti-parasite genes are activated before the parasite enters the midgut epithelium (Bahia et al., 2011; Dimopoulos et al., 2002; Dimopoulos et al., 1997). The same is shown presently using dead parasites. The simultaneous induction of AMPs in midguts and tissues of the carcass (most probably the fat body, although it is not possible to dismiss tissue attached hemocytes) suggests as yet unidentified regulatory midgut-originated mechanisms operating at distance, both during primary and secondary immune responses. Hence, the current results underscore the participation of the mosquito midgut in the innate immune response to microorganisms.

Although it was previously adduced that a persistent activation of the immune system could explain long lasting prophylactic immune responses (Sadd and Schmid-Hempel, 2006), evolutionary ecology argues that the 
costs and necessary trade-offs implied makes this an unlikely possibility (Moret and Siva-Jothy, 2003; Roth et al., 2009). On the other hand, despite the increased mobilization and reduced mitosis documented for circulating granulocytes (with a mitotic index of $0.63 \%$ ) of E. coli-infected An. gambiae (King and Hillyer, 2013), invertebrates lack the immune cell clonal expansion existing in vertebrates. We previously reported that de novo DNA synthesis occurs in mosquitoes (Hernandez-Martinez et al., 2013; Hernandez-Martinez et al., 2006), evidenced by the incorporation of BrdU and activation of PCNA in the tissues of these insects after an immune challenge. Such synthesis is most probably by endoreplication, as no mitotic cells have been observed.

In the present study we documented an enhanced DNA synthesis after the second exposure to the same pathogen in primed mosquitoes. Therefore, we explored the possibility that in this immune response an important role was played by one of the key factors in cell cycle switching towards endoreplication-hnt. In all eukaryotic cells and in Drosophila, hnt (a zinc-finger protein) is a key factor that mediates the participation of Notch in the switching of the cell cycle from mitosis to the endocycle (Sun and Deng, 2007). This scenario implies that midgut cells have an increased number of copies of the genes required for a quick and effective response, which may include the fast production of effector transcripts and proteins.

In the present An. albimanus model, the biphasic AMP mRNA expression indicates that the mosquito immune activation was not sustained. Rather, the primed stage was established as early as seven days after the first parasite exposure, meaning that the immune response had already returned to basal levels and was reinduced at higher levels by the second exposure to Plasmodium. This also indicates the presence of a not yet identified mechanism to establish a stand-by specific immune preparedness (priming) that it is activated only after a reencounter with the same inducing pathogen. However, further investigation is required into the precise mechanisms that render the mosquito better prepared to mount an immune response (priming) once the inducing pathogen is neutralized (Hauton and Smith, 2007; Little and Kraaijeveld, 2004).

In summary, we have documented that priming in An. albimanus by a $P$. berghei infection has at least two characteristics of an adaptive immune response - long duration (Contreras-Garduno et al., 2014) and a biphasic dynamic. The condition of specificity requires highly specialized effector molecules and may not be necessary for organisms that produce multi-target effector molecules. To resolve this question, further research 
is required on the possible mechanisms of the mosquito immune response to bacteria and malaria parasites. In this sense, the increase in transcription for $h n t$ is not only indicative of crucial modifications during the priming response to an immune challenge in the midgut cells, but also suggests a potential molecular mechanism for the generation of a primed state in preparedness for further immune challenges. In this scenario, midgut cells would have an increased number of copies of genes required for a quick and effective response, which may include the fast production of effector transcripts and proteins. These results warrant further analysis of this cell cycle switching as a potential non-proliferative mechanism participating in the immune priming response in the mosquito midgut. The identification of mechanisms and molecules responsible for priming should certainly provide insights into the evolution of immune memory and contribute to advances in the proposed genetic engineering strategies to interrupt diseases transmitted by invertebrate vectors. Even though there is accumulating evidence of an evolutionary convergence linking invertebrate priming and immune memory, further studies are needed in this sense.

\section{Acknowledgments}

We are grateful for the financial support from the Bill and Melinda Gates Foundation (Grant \# CI:783). MCRG is thankful to CONACyT for grant \#107006. The authors thank the personnel from the insectary for providing mosquitoes and Allan Larsen for proofreading the manuscript.

\section{Footnotes}

Figure 1. Immune priming with $P$. berghei in An. albimanus treated and untreated with antibiotics. Both groups of mosquitoes primed with gametocytes of $P$. berghei ANKA 2.34 (treated and untreated with antibiotics) showed fewer oocysts in their midgut compared to the unprimed control group (treated and untreated with 
antibiotics). The lowest mean number of oocysts was observed in primed mosquitoes treated with antibiotics.

Values are expressed as the mean $\pm \mathrm{SE}$.

Figure 2. Immune priming with gametocytes of P. berghei ANKA 2.34 and P. berghei DKo in An. albimanus untreated with antibiotics. Fewer oocysts were detected in mosquitoes primed with DKo parasites compared to those primed with gametocytes of P. berghei ANKA 2.34, and both these primed groups developed fewer oocysts than the unprimed control group. Values are expressed as the mean $\pm \mathrm{SE}$.

Figure 3. After immune priming with live and dead ookinetes of P. berghei ANKA 2.34 in An. albimanus previously treated with antibiotics, both groups of primed mosquitoes developed fewer oocysts than the unprimed control group. Values are expressed as the mean $\pm \mathrm{SE}$.

Figure 4. Analysis of antimicrobial peptide (AMP) expression in primed An. albimanus mosquitoes (exposed to P. berghei gametocytes) demonstrated a biphasic response of AMP expression in midguts (A) and abdominal tissues (B), which was not affected by the antibiotic treatment. RT-PCR analysis showed that the relative AMP mRNA levels increased in both the midgut and abdomen during the first exposure to P. berghei (priming), decreased to basal levels (by the $7^{\text {th }}$ day post-infection), and increased again after the second exposure (challenge) to higher levels than those observed with the first parasite encounter. The primed group is represented with continuous lines and the unprimed control group with dotted lines.

Figure 5. A) Immunofluorescence of BrdU incorporation in gut samples from primed and unprimed $A n$. albimanus mosquitoes. Before priming (day 0) there were few nuclei with the fluorescent label of BrdU in either the primed or unprimed groups. At eight days post-priming, most samples showed strong BrdU incorporation in the primed versus unprimed group. At twelve days post-priming, most gut samples (whether primed or unprimed) showed BrdU incorporation into the nuclei. B) Relative quantification of BrdU incorporation in primed and unprimed gut samples after the $P$. berghei challenge (second exposure). BrdU 
incorporated in DNA was estimated by absorbance levels in an ELISA assay. Values are expressed as the mean $\pm \operatorname{SEM}(n=2)$ of the relative-increase in BrdU incorporation in the primed group compared to the control group. Only at $24 \mathrm{~h}$ after challenge (day 8) were higher levels of BrdU found in primed versus unprimed gut samples. C) Expression of $h n t$ in mosquito midguts treated with antibiotics at $72 \mathrm{~h}$ after both priming and the challenge with P. berghei parasites. After An. albimanus were fed with live ookinetes during the second exposure (challenge), greater hnt expression was found in the midgut of mosquitoes previously fed with gametocytes (primed) compared to those previously fed with normal blood (unprimed). *significant differences at $p<0.0001$. Bar $=200 \mu \mathrm{m}$.

\section{References}

Aguilar, R., Jedlicka, A.E., Mintz, M., Mahairaki, V., Scott, A.L., Dimopoulos, G., 2005. Global gene expression analysis of Anopheles gambiae responses to microbial challenge. Insect biochemistry and molecular biology 35, 709-719.

Akira, S., Takeda, K., Kaisho, T., 2001. Toll-like receptors: critical proteins linking innate and acquired immunity. Nature immunology 2, 675-680. 
Bahia, A.C., Kubota, M.S., Tempone, A.J., Araujo, H.R., Guedes, B.A., Orfano, A.S., Tadei, W.P., RiosVelasquez, C.M., Han, Y.S., Secundino, N.F., Barillas-Mury, C., Pimenta, P.F., Traub-Cseko, Y.M., 2011. The JAK-STAT pathway controls Plasmodium vivax load in early stages of Anopheles aquasalis infection. PLoS neglected tropical diseases 5, e1317.

Baton, L.A., Robertson, A., Warr, E., Strand, M.R., Dimopoulos, G., 2009. Genome-wide transcriptomic profiling of Anopheles gambiae hemocytes reveals pathogen-specific signatures upon bacterial challenge and Plasmodium berghei infection. BMC genomics 10, 257.

Blandin, S., Shiao, S.H., Moita, L.F., Janse, C.J., Waters, A.P., Kafatos, F.C., Levashina, E.A., 2004.

Complement-like protein TEP1 is a determinant of vectorial capacity in the malaria vector Anopheles gambiae. Cell 116, 661-670.

Boman, H.G., Steiner, H., 1981. Humoral immunity in Cecropia pupae. Current topics in microbiology and immunology 94-95, 75-91.

Bravo, R., Frank, R., Blundell, P.A., Macdonald-Bravo, H., 1987. Cyclin/PCNA is the auxiliary protein of DNA polymerase-delta. Nature 326, 515-517.

Brehélin, M., Roch, P., 2008. Specificity, learning and memory in the innate immune response. Invertebr Surv J 5, 103-109.

Cirimotich, C.M., Dong, Y., Clayton, A.M., Sandiford, S.L., Souza-Neto, J.A., Mulenga, M., Dimopoulos, G., 2011. Natural microbe-mediated refractoriness to Plasmodium infection in Anopheles gambiae. Science 332, 855-858.

Contreras-Garduno, J., Rodriguez, M.C., Rodriguez, M.H., Alvarado-Delgado, A., Lanz-Mendoza, H., 2014. Cost of immune priming within generations: trade-off between infection and reproduction. Microbes and infection / Institut Pasteur 16, 261-267.

Costa, A., Jan, E., Sarnow, P., Schneider, D., 2009. The Imd pathway is involved in antiviral immune responses in Drosophila. PloS one 4, e7436.

Chan, A.S., Rodriguez, M.H., Torres, J.A., Rodriguez Mdel, C., Villarreal, C., 1994. Susceptibility of three laboratory strains of Anopheles albimanus (Diptera: Culicidae) to coindigenous Plasmodium vivax in southern Mexico. Journal of medical entomology 31, 400-403.

Chen, C.C., Laurence, B.R., 1985. An ultrastructural study on the encapsulation of microfilariae of Brugia pahangi in the haemocoel of Anopheles quadrimaculatus. International journal for parasitology 15, 421-428. De Gregorio, E., Spellman, P.T., Tzou, P., Rubin, G.M., Lemaitre, B., 2002. The Toll and Imd pathways are the major regulators of the immune response in Drosophila. The EMBO journal 21, 2568-2579.

Dimopoulos, G., Christophides, G.K., Meister, S., Schultz, J., White, K.P., Barillas-Mury, C., Kafatos, F.C., 2002. Genome expression analysis of Anopheles gambiae: responses to injury, bacterial challenge, and malaria infection. Proceedings of the National Academy of Sciences of the United States of America 99, 8814-8819. Dimopoulos, G., Muller, H.M., Levashina, E.A., Kafatos, F.C., 2001. Innate immune defense against malaria infection in the mosquito. Current opinion in immunology 13, 79-88.

Dimopoulos, G., Richman, A., Muller, H.M., Kafatos, F.C., 1997. Molecular immune responses of the mosquito Anopheles gambiae to bacteria and malaria parasites. Proceedings of the National Academy of Sciences of the United States of America 94, 11508-11513.

Dimopoulos, G., Seeley, D., Wolf, A., Kafatos, F.C., 1998. Malaria infection of the mosquito Anopheles gambiae activates immune-responsive genes during critical transition stages of the parasite life cycle. The EMBO journal 17, 6115-6123.

Dong, Y., Aguilar, R., Xi, Z., Warr, E., Mongin, E., Dimopoulos, G., 2006a. Anopheles gambiae immune responses to human and rodent Plasmodium parasite species. PLoS pathogens 2, e52.

Dong, Y., Dimopoulos, G., 2009. Anopheles fibrinogen-related proteins provide expanded pattern recognition capacity against bacteria and malaria parasites. The Journal of biological chemistry 284, 9835-9844.

Dong, Y., Manfredini, F., Dimopoulos, G., 2009. Implication of the mosquito midgut microbiota in the defense against malaria parasites. PLoS pathogens 5, e1000423.

Dong, Y., Taylor, H.E., Dimopoulos, G., 2006b. AgDscam, a hypervariable immunoglobulin domaincontaining receptor of the Anopheles gambiae innate immune system. PLoS biology 4, e229.

Eyles, D.E., 1950. A stain for malarial oocysts in temporary preparations. The Journal of parasitology 36, 501. 
Franke-Fayard, B., Trueman, H., Ramesar, J., Mendoza, J., van der Keur, M., van der Linden, R., Sinden, R.E., Waters, A.P., Janse, C.J., 2004. A Plasmodium berghei reference line that constitutively expresses GFP at a high level throughout the complete life cycle. Molecular and biochemical parasitology 137, 23-33.

Garver, L.S., Bahia, A.C., Das, S., Souza-Neto, J.A., Shiao, J., Dong, Y., Dimopoulos, G., 2012. Anopheles Imd pathway factors and effectors in infection intensity-dependent anti-Plasmodium action. PLoS pathogens 8 , e1002737.

Garver, L.S., de Almeida Oliveira, G., Barillas-Mury, C., 2013. The JNK pathway is a key mediator of Anopheles gambiae antiplasmodial immunity. PLoS pathogens 9, e1003622.

Garver, L.S., Dong, Y., Dimopoulos, G., 2009. Caspar controls resistance to Plasmodium falciparum in diverse anopheline species. PLoS pathogens 5, e1000335.

Gonzalez-Ceron, L., Santillan, F., Rodriguez, M.H., Mendez, D., Hernandez-Avila, J.E., 2003. Bacteria in midguts of field-collected Anopheles albimanus block Plasmodium vivax sporogonic development. Journal of medical entomology 40, 371-374.

Gupta, L., Molina-Cruz, A., Kumar, S., Rodrigues, J., Dixit, R., Zamora, R.E., Barillas-Mury, C., 2009. The STAT pathway mediates late-phase immunity against Plasmodium in the mosquito Anopheles gambiae. Cell host \& microbe 5, 498-507.

Hauton, C., Smith, V.J., 2007. Adaptive immunity in invertebrates: a straw house without a mechanistic foundation. BioEssays : news and reviews in molecular, cellular and developmental biology 29, 1138-1146. Hernandez-Martinez, S., Barradas-Bautista, D., Rodriguez, M.H., 2013. Diferential DNA synthesis in Anopheles albimanus tissues induced by immune challenge with different microorganisms. Archives of insect biochemistry and physiology 84, 1-14.

Hernandez-Martinez, S., Lanz, H., Rodriguez, M.H., Gonzalez-Ceron, L., Tsutsumi, V., 2002. Cellularmediated reactions to foreign organisms inoculated into the hemocoel of Anopheles albimanus (Diptera: Culicidae). Journal of medical entomology 39, 61-69.

Hernandez-Martinez, S., Roman-Martinez, U., Martinez-Barnetche, J., Garrido, E., Rodriguez, M.H., LanzMendoza, H., 2006. Induction of DNA synthesis in Anopheles albimanus tissue cultures in response to a Saccharomyces cerevisiae challenge. Archives of insect biochemistry and physiology 63, 147-158.

Herrera-Ortiz, A., Martinez-Barnetche, J., Smit, N., Rodriguez, M.H., Lanz-Mendoza, H., 2011. The effect of nitric oxide and hydrogen peroxide in the activation of the systemic immune response of Anopheles albimanus infected with Plasmodium berghei. Developmental and comparative immunology 35, 44-50.

Hillyer, J.F., Barreau, C., Vernick, K.D., 2007. Efficiency of salivary gland invasion by malaria sporozoites is controlled by rapid sporozoite destruction in the mosquito haemocoel. International journal for parasitology 37 , 673-681.

Hoffmann, J.A., Hoffmann, D., 1990. The inducible antibacterial peptides of dipteran insects. Research in immunology 141, 910-918.

King, J.G., Hillyer, J.F., 2013. Spatial and temporal in vivo analysis of circulating and sessile immune cells in mosquitoes: hemocyte mitosis following infection. BMC biology 11, 55.

Kurtz, J., 2005. Specific memory within innate immune systems. Trends in immunology 26, 186-192.

Kurtz, J., Franz, K., 2003. Innate defence: evidence for memory in invertebrate immunity. Nature 425, 37-38. Little, T.J., Kraaijeveld, A.R., 2004. Ecological and evolutionary implications of immunological priming in invertebrates. Trends in ecology \& evolution 19, 58-60.

Marois, E., 2011. The multifaceted mosquito anti-Plasmodium response. Current opinion in microbiology 14, 429-435.

Martinez-Barnetche, J., Gomez-Barreto, R.E., Ovilla-Munoz, M., Tellez-Sosa, J., Garcia Lopez, D.E., Dinglasan, R.R., Ubaida Mohien, C., MacCallum, R.M., Redmond, S.N., Gibbons, J.G., Rokas, A., Machado, C.A., Cazares-Raga, F.E., Gonzalez-Ceron, L., Hernandez-Martinez, S., Rodriguez Lopez, M.H., 2012.

Transcriptome of the adult female malaria mosquito vector Anopheles albimanus. BMC genomics 13, 207.

Medzhitov, R., Janeway, C.A., Jr., 2002. Decoding the patterns of self and nonself by the innate immune system. Science 296, 298-300.

Meister, S., Kanzok, S.M., Zheng, X.L., Luna, C., Li, T.R., Hoa, N.T., Clayton, J.R., White, K.P., Kafatos, F.C., Christophides, G.K., Zheng, L., 2005. Immune signaling pathways regulating bacterial and malaria parasite 
infection of the mosquito Anopheles gambiae. Proceedings of the National Academy of Sciences of the United States of America 102, 11420-11425.

Michel, K., Kafatos, F.C., 2005. Mosquito immunity against Plasmodium. Insect biochemistry and molecular biology 35, 677-689.

Moret, Y., Siva-Jothy, M.T., 2003. Adaptive innate immunity? Responsive-mode prophylaxis in the mealworm beetle, Tenebrio molitor. Proceedings. Biological sciences / The Royal Society 270, 2475-2480.

Ng, T.H., Chiang, Y.A., Yeh, Y.C., Wang, H.C., 2014. Review of Dscam-mediated immunity in shrimp and other arthropods. Developmental and comparative immunology 46, 129-138.

Noden, B.H., Vaughan, J.A., Pumpuni, C.B., Beier, J.C., 2011. Mosquito ingestion of antibodies against mosquito midgut microbiota improves conversion of ookinetes to oocysts for Plasmodium falciparum, but not P. yoelii. Parasitology international 60, 440-446.

Oduol, F., Xu, J., Niare, O., Natarajan, R., Vernick, K.D., 2000. Genes identified by an expression screen of the vector mosquito Anopheles gambiae display differential molecular immune response to malaria parasites and bacteria. Proceedings of the National Academy of Sciences of the United States of America 97, 11397-11402. Pham, L.N., Dionne, M.S., Shirasu-Hiza, M., Schneider, D.S., 2007. A specific primed immune response in Drosophila is dependent on phagocytes. PLoS pathogens 3, e26.

Pike, A., Vadlamani, A., Sandiford, S.L., Gacita, A., Dimopoulos, G., 2014. Characterization of the Rel2regulated transcriptome and proteome of Anopheles stephensi identifies new anti-Plasmodium factors. Insect biochemistry and molecular biology 52, 82-93.

Pumpuni, C.B., Demaio, J., Kent, M., Davis, J.R., Beier, J.C., 1996. Bacterial population dynamics in three anopheline species: the impact on Plasmodium sporogonic development. The American journal of tropical medicine and hygiene 54, 214-218.

Rodrigues, J., Brayner, F.A., Alves, L.C., Dixit, R., Barillas-Mury, C., 2010. Hemocyte differentiation mediates innate immune memory in Anopheles gambiae mosquitoes. Science 329, 1353-1355.

Rodriguez, M.C., Margos, G., Compton, H., Ku, M., Lanz, H., Rodriguez, M.H., Sinden, R.E., 2002.

Plasmodium berghei: routine production of pure gametocytes, extracellular gametes, zygotes, and ookinetes.

Experimental parasitology 101, 73-76.

Rolff, J., Siva-Jothy, M.T., 2003. Invertebrate ecological immunology. Science 301, 472-475.

Roth, O., Sadd, B.M., Schmid-Hempel, P., Kurtz, J., 2009. Strain-specific priming of resistance in the red flour beetle, Tribolium castaneum. Proceedings. Biological sciences / The Royal Society 276, 145-151.

Sadd, B.M., Schmid-Hempel, P., 2006. Insect immunity shows specificity in protection upon secondary pathogen exposure. Current biology : CB 16, 1206-1210.

Sambrook, J., Russell, D.W., 2001. Molecular cloning : a laboratory manual, 3rd ed. Cold Spring Harbor Laboratory Press, Cold Spring Harbor, N.Y.

Sanders, H.R., Foy, B.D., Evans, A.M., Ross, L.S., Beaty, B.J., Olson, K.E., Gill, S.S., 2005. Sindbis virus induces transport processes and alters expression of innate immunity pathway genes in the midgut of the disease vector, Aedes aegypti. Insect biochemistry and molecular biology 35, 1293-1307.

Schulenburg, H., Boehnisch, C., Michiels, N.K., 2007. How do invertebrates generate a highly specific innate immune response? Molecular immunology 44, 3338-3344.

Smith, P.H., Mwangi, J.M., Afrane, Y.A., Yan, G., Obbard, D.J., Ranford-Cartwright, L.C., Little, T.J., 2011. Alternative splicing of the Anopheles gambiae Dscam gene in diverse Plasmodium falciparum infections. Malaria journal 10, 156.

Sun, J., Deng, W.M., 2007. Hindsight mediates the role of notch in suppressing hedgehog signaling and cell proliferation. Developmental cell 12, 431-442.

Tahar, R., Boudin, C., Thiery, I., Bourgouin, C., 2002. Immune response of Anopheles gambiae to the early sporogonic stages of the human malaria parasite Plasmodium falciparum. The EMBO journal 21, 6673-6680. Tomas, A.M., Margos, G., Dimopoulos, G., van Lin, L.H., de Koning-Ward, T.F., Sinha, R., Lupetti, P., Beetsma, A.L., Rodriguez, M.C., Karras, M., Hager, A., Mendoza, J., Butcher, G.A., Kafatos, F., Janse, C.J., Waters, A.P., Sinden, R.E., 2001. P25 and P28 proteins of the malaria ookinete surface have multiple and partially redundant functions. The EMBO journal 20, 3975-3983.

Vlachou, D., Schlegelmilch, T., Christophides, G.K., Kafatos, F.C., 2005. Functional genomic analysis of midgut epithelial responses in Anopheles during Plasmodium invasion. Current biology : CB 15, 1185-1195. 
Warr, E., Das, S., Dong, Y., Dimopoulos, G., 2008. The Gram-negative bacteria-binding protein gene family: its role in the innate immune system of anopheles gambiae and in anti-Plasmodium defence. Insect molecular biology 17, 39-51.

Waterhouse, R.M., Kriventseva, E.V., Meister, S., Xi, Z., Alvarez, K.S., Bartholomay, L.C., Barillas-Mury, C., Bian, G., Blandin, S., Christensen, B.M., Dong, Y., Jiang, H., Kanost, M.R., Koutsos, A.C., Levashina, E.A., Li, J., Ligoxygakis, P., Maccallum, R.M., Mayhew, G.F., Mendes, A., Michel, K., Osta, M.A., Paskewitz, S., Shin, S.W., Vlachou, D., Wang, L., Wei, W., Zheng, L., Zou, Z., Severson, D.W., Raikhel, A.S., Kafatos, F.C., Dimopoulos, G., Zdobnov, E.M., Christophides, G.K., 2007. Evolutionary dynamics of immune-related genes and pathways in disease-vector mosquitoes. Science 316, 1738-1743.

Winger, L.A., Tirawanchai, N., Nicholas, J., Carter, H.E., Smith, J.E., Sinden, R.E., 1988. Ookinete antigens of Plasmodium berghei. Appearance on the zygote surface of an $\mathrm{Mr} 21 \mathrm{kD}$ determinant identified by transmissionblocking monoclonal antibodies. Parasite immunology 10, 193-207.

Witteveldt, J., Cifuentes, C.C., Vlak, J.M., van Hulten, M.C., 2004. Protection of Penaeus monodon against white spot syndrome virus by oral vaccination. Journal of virology 78, 2057-2061. 


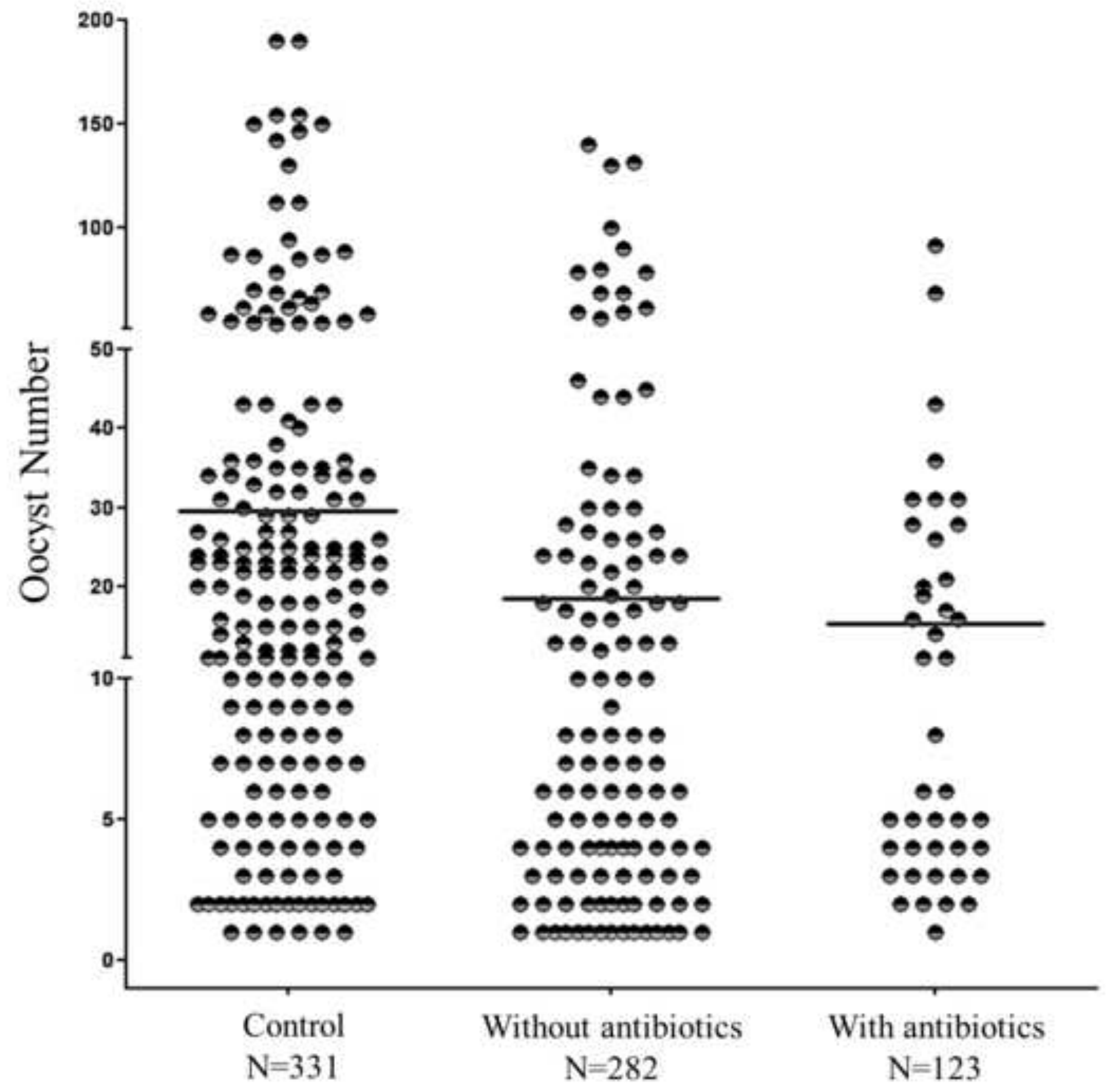

$\mathrm{N}-282$

$\mathrm{N}=123$ 


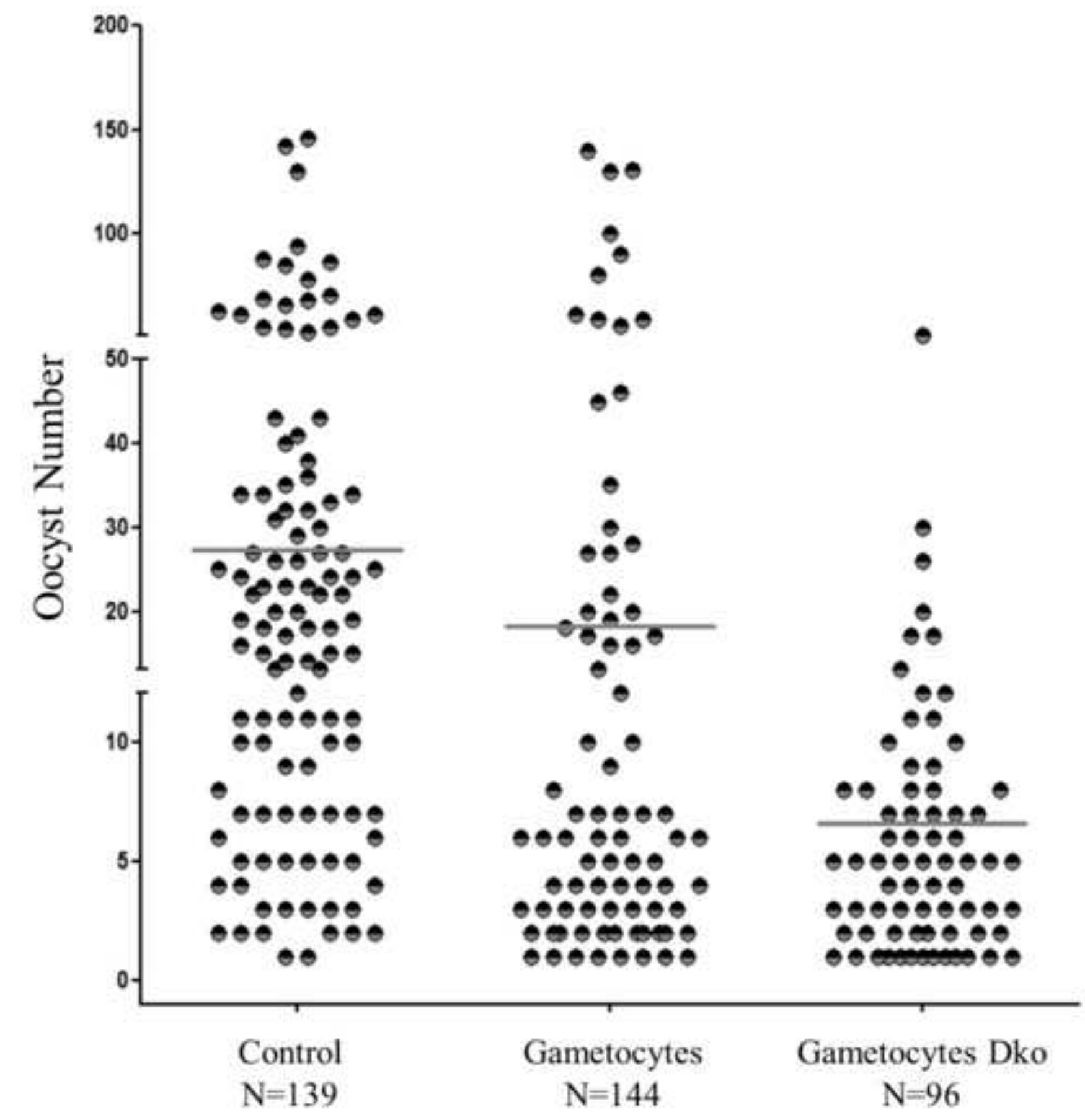




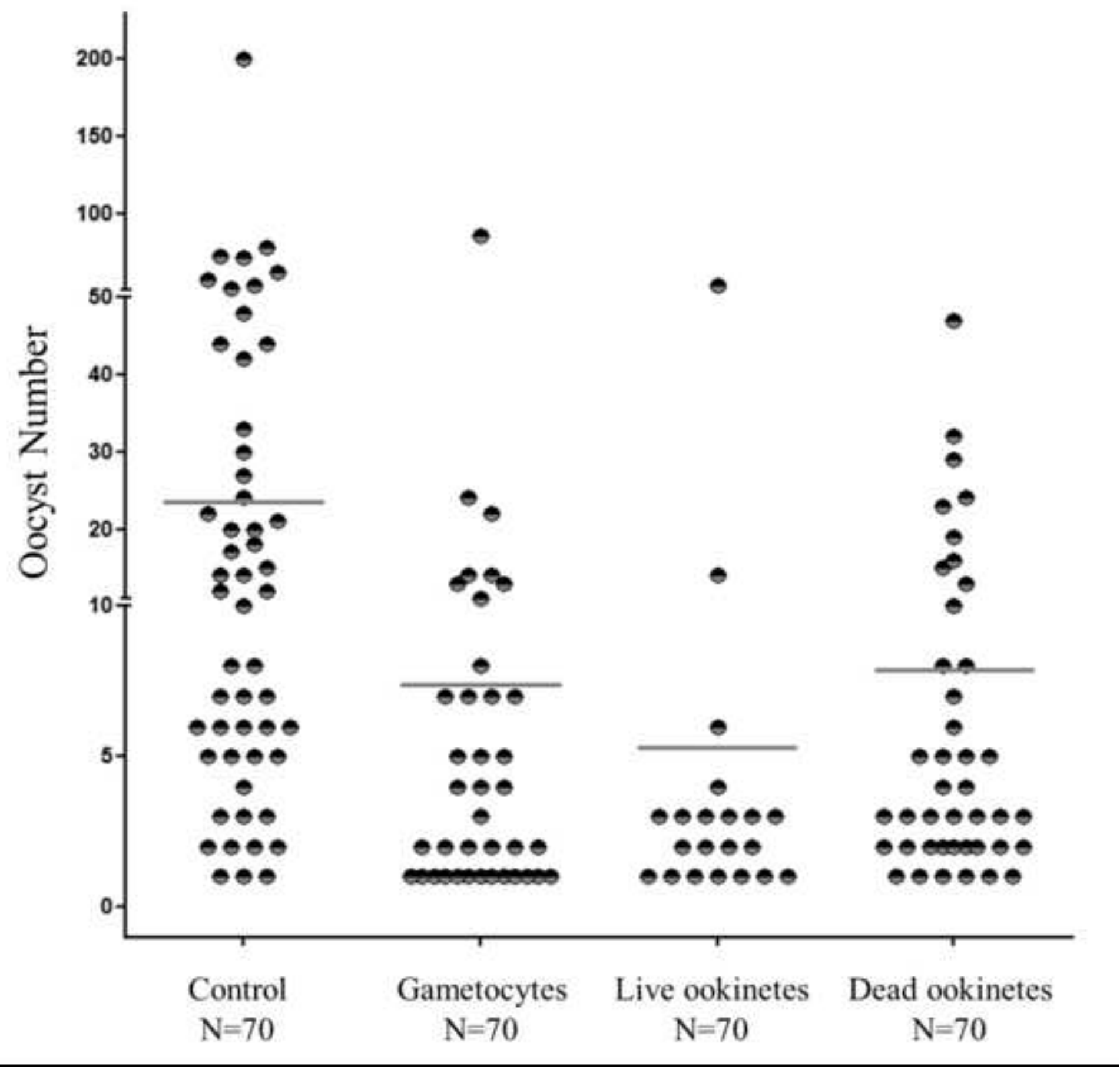



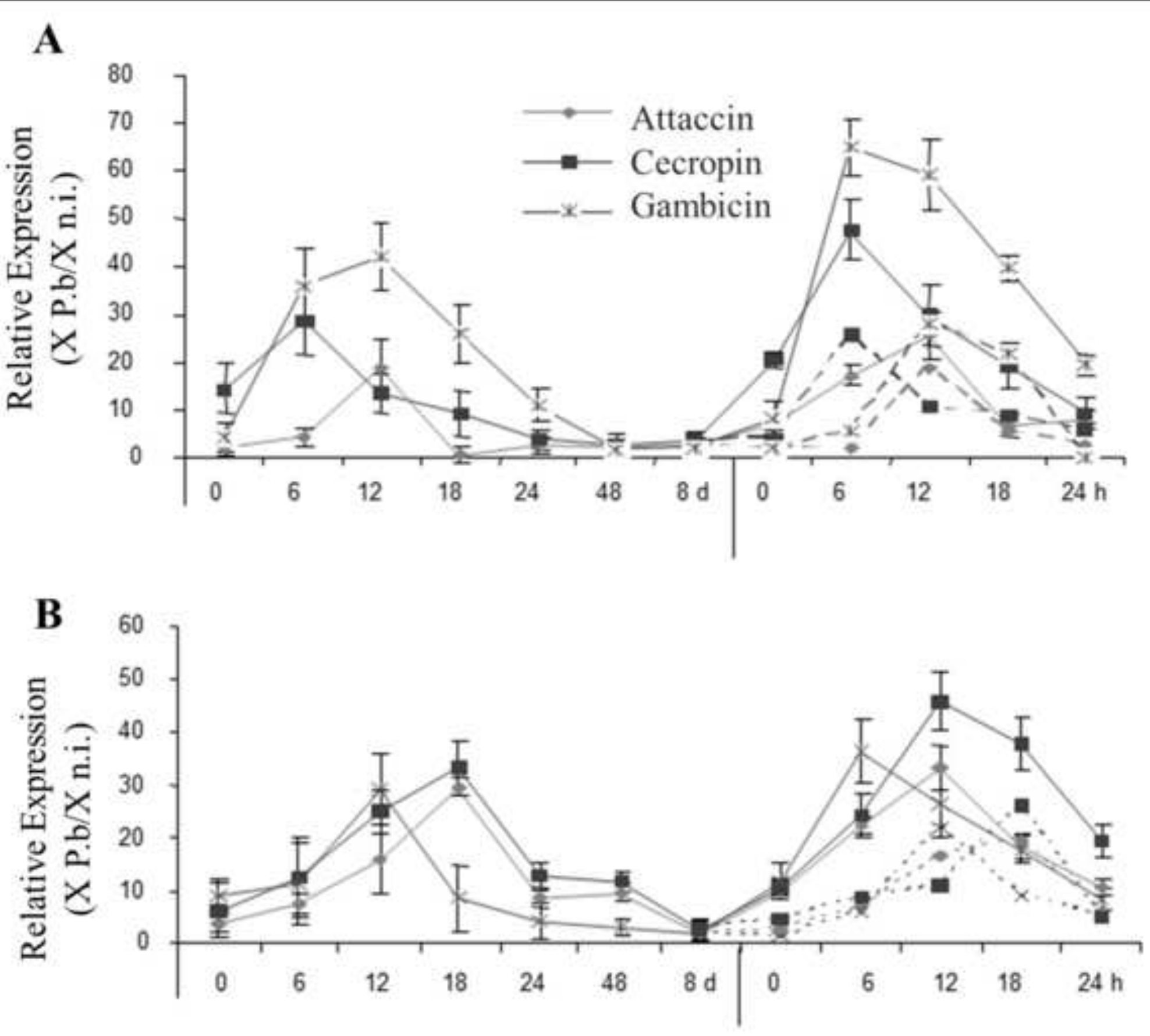


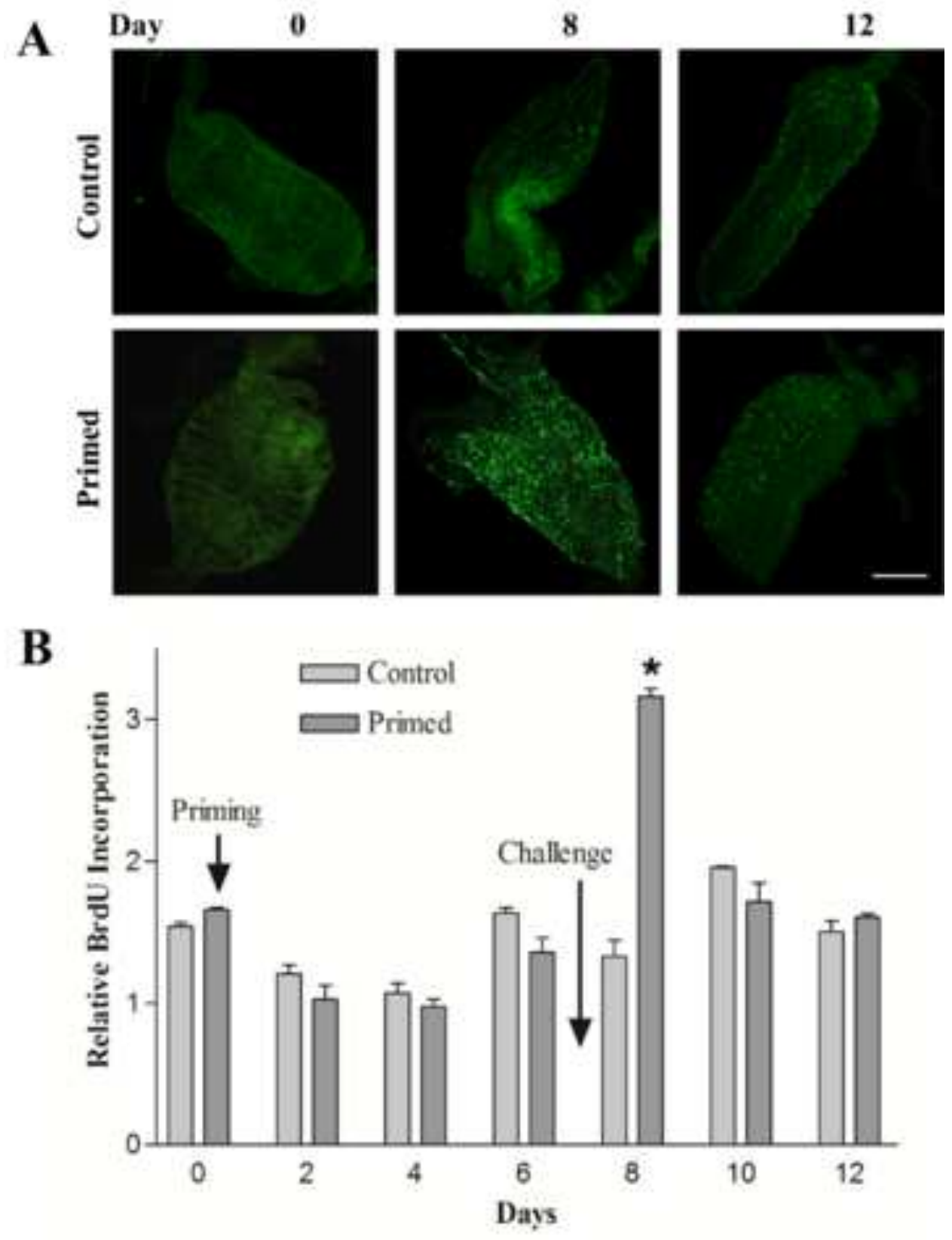

C

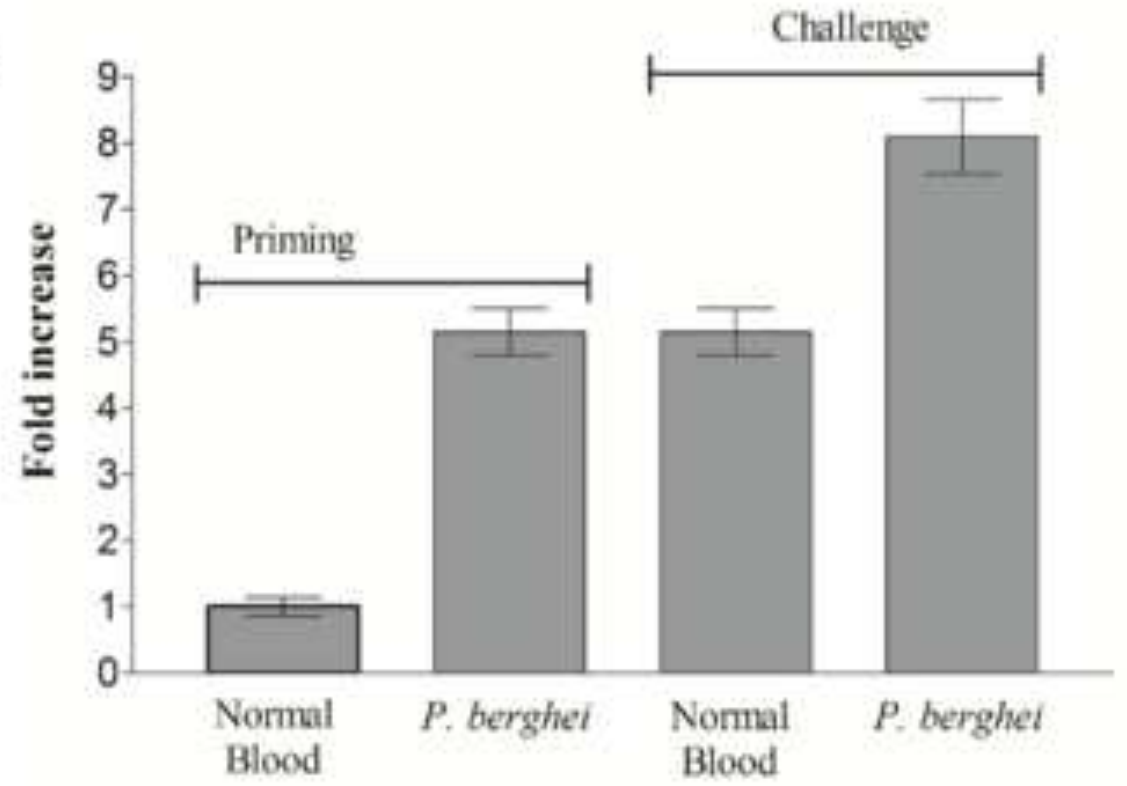

